\title{
Conversational Sales Assistant for Online Shopping
}

\author{
Margo Budzikowska, Joyce Chai, Sunil Govindappa, Veronika Horvath, Nanda Kambhatla, \\ Nicolas Nicolov \& Wlodek Zadrozny \\ Conversational Machines Group \\ IBM T. J. Watson Research Center \\ 30 Saw Mill River Rd, Hawthorne, NY 10532, U.S.A. \\ \{sm1, jchai, govindap, veronika, nanda, nicolas,wlodz\}@us.ibm.com
}

\begin{abstract}
Websites of businesses should accommodate both customer needs and business requirements. Traditional menu-driven navigation and key word search do not allow users to describe their intentions precisely. We have developed a conversational interface to online shopping that provides convenient, personalized access to information using natural language dialog. User studies show significantly reduced length of interactions in terms of time and number of clicks in finding products. The core dialog engine is easily adaptable to other domains.
\end{abstract}

\section{INTRODUCTION}

Natural language dialog has been used in many areas, such as for call-center/routing application (Carpenter \& Chu-Carroll 1998), email routing (Walker, Fromer \& Narayanan 1998), information retrieval and database access (Androutsopoulos \& Ritchie 1995), and for telephony banking (Zadrozny et al. 1998). In this demonstration, we present a natural language dialog interface to online shopping. Our user studies show natural language dialog to be a very effective means for negotiating user's requests and intentions in this domain.

\section{SYSTEM ARCHITECTURE}

In our system, a presentation manager captures queries from users, employs a parser to transform the user's query into a logical form, and sends the logical form to a dialog manager. The presentation manager is also responsible for obtaining the system's response from the dialog manager and presenting it to the user using template-based generation. The dialog manager formulates action plans for an action manager to perform backend tasks such as database access, business transactions, etc. The dialog manager applies information state-based dialog strategies to formulate responses depending on the current state, discourse history and the action results from the action manager.
The Data Management Subsystem maintains a "concept" repository with common sense "concepts" and a phrasal lexicon that lists possible ways for referring to the concepts. Business Rules map concepts to business specifications by defining concepts using a propositional logic formula of constraints over product specifications. Thus, the Business Rules reflect business goals and decisions. The Extended Database combines product specifications and precompiled evaluations of the concept definitions for each product to provide a representation that guides the natural language dialog. We are investigating automated tools for helping developers and maintainers extract relevant concepts and terms on the basis of user descriptions and queries about products.

\section{EVALUATION}

We conducted several user studies to evaluate the usability of NLA (Chai et al. 2000). In one study, seventeen test subjects preferred the dialog-driven navigation of NLA two to one over menu-driven navigation. Moreover, with NLA, the average number of clicks was reduced by $63.2 \%$ and the average time was reduced by $33.3 \%$. Analysis of the user queries (average length $=5.31$ words long; standard deviation $=2.62 ; 85 \%$ of inputs are noun phrases) revealed the brevity and relative linguistic simplicity of user input. Hence, shallow parsing techniques were adequate for processing user input. In general, sophisticated dialog management appears to be more important than the ability to handle complex natural language sentences. The user studies also highlighted the need to combine multiple modalities and styles of interaction.

\section{REFERENCES}

[1] Androutsopoulos, Ion \& Ritchie, Graeme. Natural Language Interfaces to Databases - An Introduction, Natural Language Engineering 1.1:29-81, 1995.

[2] Carpenter, Bob \& Chu-Carroll, Jeniffer. Natural Language Call Routing: A Robust, Self-organizing Approach, Proceedings of the 5th International Conference on Spoken Language Processing, 1998.

[3] Chai, J., Lin, J., Zadrozny, W., Ye, Y., Budzikowska, M., Horvath, V., Kambhatla, N. \& Wolf, C. Comparative Evaluation of a Natural Language Dialog Based System and a Menu-Driven System for Information Access: A Case Study, Proceedings of RIAO 2000, Paris, 2000. 

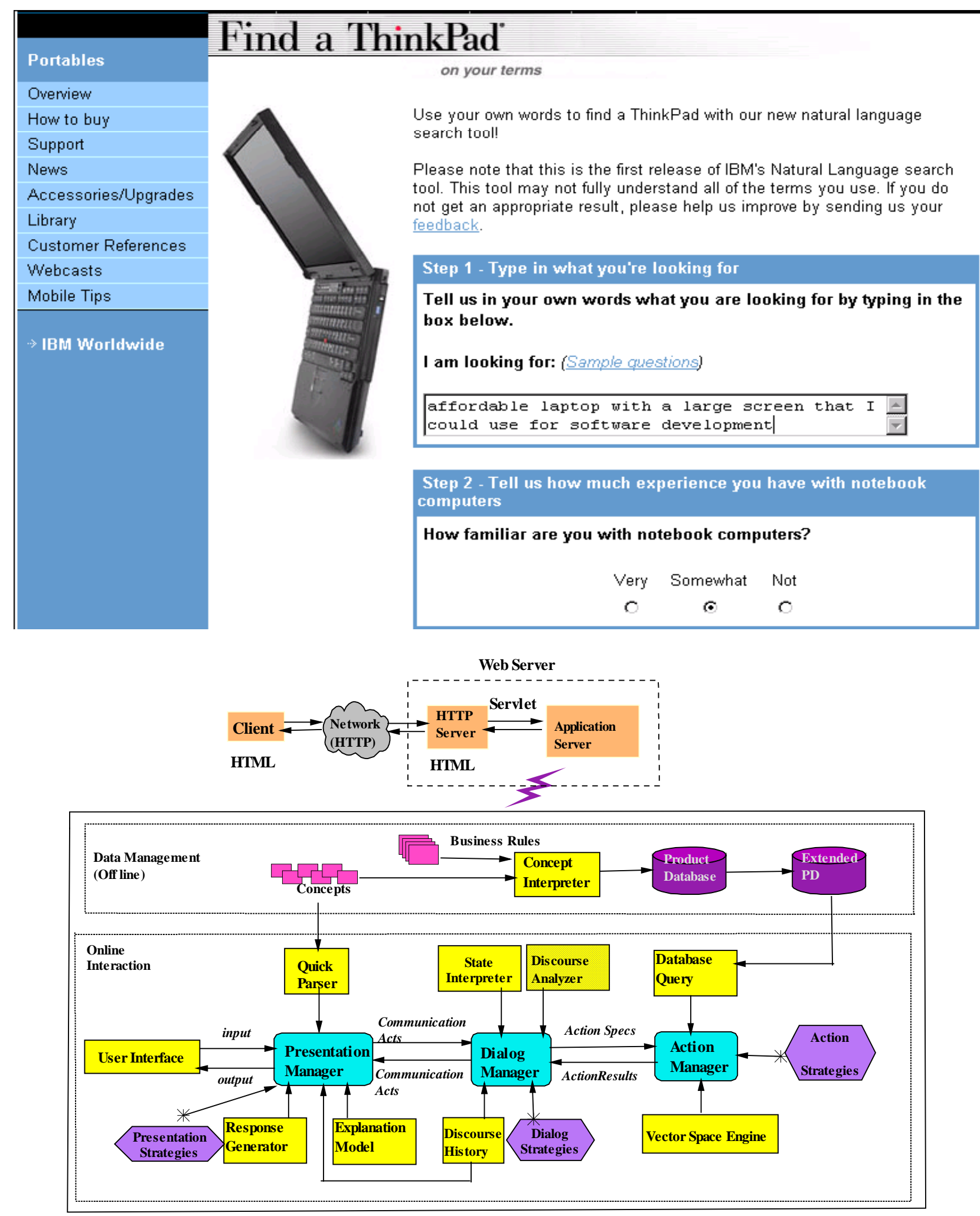

[4] Saito, M. \& Ohmura, K. A Cognitive Model for Searching for Ill-defined Targets on the Web - The Relationship between Search Strategies and User Satisfaction. 21st Int. Conf. on Research and Development in Information Retrieval, Australia, 1998.

[5] Walker, M., Fromer, J. \& Narayanan, S. Learning Optimal Dialogue Strategies: A Case Study of a Spoken Dialogue
Agent for Email, 36th Annual Meeting of the ACL, Montreal, Canada, 1998.

[6] Zadrozny, W., Wolf, C., Kambhatla, N. \& Ye, Y. Conversation Machines for Transaction Processing, Proceedings of AAAI / IAAI - 1998, Madison, Wisconsin, U.S.A., 1998. 